$\Rightarrow$ TUMOUR IMMUNOLOGY

\section{Partners in crime}

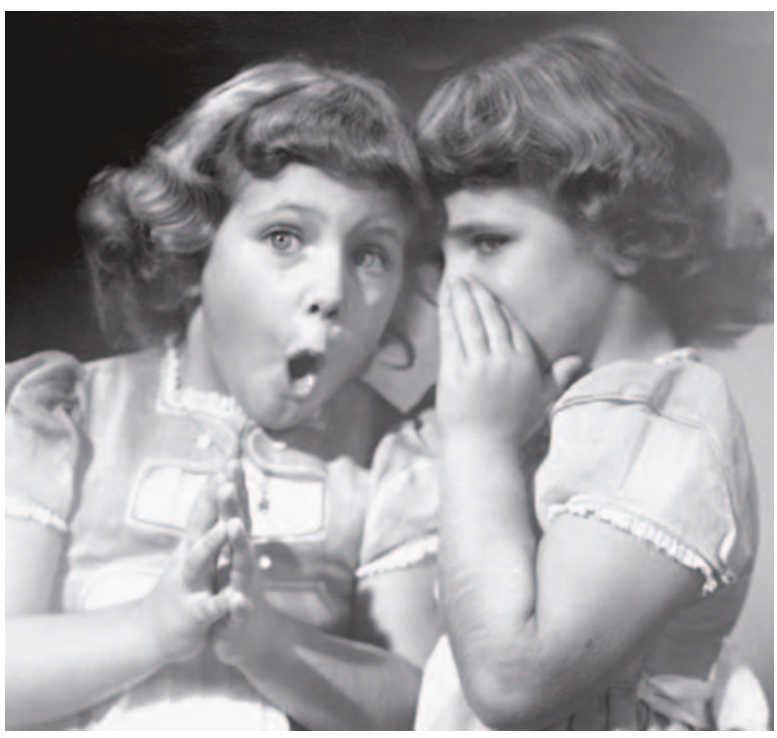

Only recently has it become apparent why manipulating the anti-tumour immune response to target established disease is often unsuccessful: the tumour and the systemic environment is largely immunosuppressive. Thomas Spies and colleagues have identified a new immunosuppressive mechanism that is mediated by a subset of $\mathrm{CD} 4^{+}$ $\mathrm{T}$ cells and by the shedding of a specific tumour antigen.

$\mathrm{T}$-cell responses are governed by a complex interaction between co-stimulatory and inhibitory ligands and their receptors. NKG2D is a receptor that can function as a co-stimulator of $\mathrm{CD}^{+} \mathrm{T}$ cells, but is not thought to be expressed by most CD4 ${ }^{+} \mathrm{T}$ cells. NKGD2 expression is downregulated by binding to MHC (major histocompatibility complex) class-I-like ligands, such as MHC class-I-related chains A and $\mathrm{B}$ (MICA and MICB). These ligands are not involved in antigen presentation, but are induced by cellular and genotoxic stress. Some tumours can shed soluble Mic ligands, and these are thought to contribute to the suppression of the $\mathrm{CD}^{+} \mathrm{T}$-cell response.

In agreement with this hypothesis, Spies and colleagues found that $\mathrm{CD}^{+} \mathrm{T}$ cells isolated from tumours and the blood of patients with Mic-expressing tumours had reduced expression of NKG2D. Surprisingly, however, they found that an increased proportion of the $\mathrm{CD} 4^{+} \mathrm{T}$ cells isolated from these patients expressed NKG2D compared with control samples (healthy individuals and patients with Mic-negative tumours).

\title{
APOPTOSIS
}

\section{A death-defying addiction}

The evasion of apoptosis, possibly through alterations in the function of the BCL2 family of pro- and anti-apoptotic proteins, is thought to be a crucial step in the oncogenic transformation of cells. Anthony Letai and colleagues have deciphered some of the specific interactions between BCL2 family members, and have identified a cellular state that they call "primed for death".

The BCL2 family of proteins can be subdivided into three groups on the basis of regions of BCL2 homology and function: multi-domain anti-apoptotic proteins (such as BCL2 and BCL$X_{L}$ ), multi-domain pro-apoptotic proteins (BAX and $\mathrm{BAK}$ ) and $\mathrm{BH} 3$-only pro-apoptotic proteins (BIM, BID and BAD, for example). The BH3-only proteins have been further subdivided into activator (BIM and BID) and sensitizer (BAD and NOXA) proteins.

Letai and colleagues used fluorescence polarization binding assays to determine selectivity in the interactions between the anti-apoptotic BCL2 proteins and a panel of oligopeptides that correspond to the $\mathrm{BH} 3$ domains of the known $\mathrm{BH} 3$-only proteins. Anti-apoptotic proteins were purified from transfected bacteria as glutathione-Stransferase fusion proteins. The proteins' binding capacity to the panel of $\mathrm{BH}_{3}$ peptides (each tagged with an $\mathrm{N}$-terminal fluorescein isothiocyanate moiety) was measured by their dissociation constant. The authors found that the anti-apoptotic family members have distinct patterns of binding affinity for specific BH3 domains. Furthermore, the binding selectivity of individual $\mathrm{BH} 3$ domains was found to correspond to their ability to antagonize the anti-apoptotic proteins in an in vitro system.

This strategy of 'BH3 profiling' was further tested in a cellular system. The prolymphocytic murine FL5.12 cell line requires interleukin 3 (IL3) for survival. IL3 withdrawal was shown to result in a dramatic increase in cellular levels of BIM, an activator BH3-only protein with a high affinity for BCL2. BIM activity renders BCL2-overexpressing FL5.12 cells wholly dependent on BCL2 when they are deprived of IL3, as BCL2 sequesters BIM and so prevents BAX oligomerization and the induction of apoptosis. So, mitochondria from FL5.12-BCL2 cells depleted of IL3 are susceptible to sensitizer $\mathrm{BH} 3$ peptides with a high affinity for BCL2 (BAD, PUMA, BMF and $B I K)$ that displace BIM from BCL2 and enable BAX oligomerization. In addition, $\mathrm{BH} 3$ profiling could distinguish cellular dependence on $\mathrm{BCL} 2$ from dependence on the related anti-apoptotic protein, MCL1.

Does this mechanism explain the role of $\mathrm{BCL} 2$ overexpression in some leukaemias? Letai and colleagues showed that leukaemia cells that overexpress $\mathrm{BCL} 2$ are sensitive to $\mathrm{BH} 3$ peptides with a high affinity for BCL2. The authors conclude that these leukaemia cells are 'primed for death', and show that these cells have high levels of BIM expression and that this is likely to explain cellular dependence on BCL2.

$\mathrm{BH} 3$ profiling is a powerful predictor of which anti-apoptotic BCL2 proteins tumour cells are likely to be dependent on. As such, this technique might be useful for predicting which of the $\mathrm{BH} 3$-mimetic drugs currently in production can be used to treat tumours that overexpress anti-apoptotic members of the BCL2 family of proteins.

Rebecca Robey, Assistant Editor, Cell Death and Differentiation

ORIGINAL RESEARCH PAPER Certo, M. et al.

Mitochondria primed by death signals determine cellular addiction to antiapoptotic BCL-2 family members. Cancer Cell 9, 351-365 (2006)

WEB SITE

Anthony Letai's lab: http://research.dfci.harvard.edu/letai 